\title{
ARTICLE FSHR ablation induces depression-like behaviors
}

\author{
Wen-kai Bi ${ }^{1,2,3,4}$, Shan-shan Shao 1,2,3,4 Zhi-wei Li $^{5}$, Yong-wei Ruan ${ }^{5}$, Si-si Luan ${ }^{1,2,3,4}$, Zhen-hua Dong ${ }^{6}$, Jing Wang ${ }^{1,2,3,4}$, \\ Shan-shan $\mathrm{Wu}^{1,2,3,4}$, Tian Guo ${ }^{1,2,3,4}$, Shi-zhan Ma ${ }^{1,2,3,4}$, Ling Gao ${ }^{1,2,3}$, Jia-jun Zhao ${ }^{1,2,3,7}$ and Zhao He $\mathrm{H}^{1,2,3,4,7}$
}

Alteration in reproductive hormones profile is associated with the increasing risk of menopausal depression in women. Serum follicle-stimulating hormone (FSH) level is changed during the menopause transition, while the effect of FSH on menopausal depression has remained undefined. In this study we investigated whether or how FSH affected menopausal depression in postmenopausal (ovariectomized) FSHR knockout mice $\left(F \mathrm{shr}^{-1-}\right)$. We found that $F s r^{-1-}$ mice displayed aggravated depression-like behaviors, accompanied by severe oxidative stress in the whole brain, resulted from significantly reduced glutamate cysteine ligase modifier subunit (GCLm) in glutathione synthesis and glucose-6-phosphate dehydrogenase (G6PD) in NADP/NADPH transition. Importantly, administration of ROS scavenger $\mathrm{N}$-acetyl cysteine (NAC, $150 \mathrm{mg} \cdot \mathrm{kg}^{-1} \cdot \mathrm{d}^{-1}$, i.p. for 12 weeks) attenuated the depression-like behaviors of $\mathrm{Fshr}^{-1-}$ mice. Consistent with these in vivo experiment results, we found that pretreatment with FSH $(50,100 \mathrm{ng} / \mathrm{mL})$ dose-dependently increased protein levels of GCLm and G6PD, and decreased the ROS production in N2a mouse neuroblastoma cells. These findings demonstrate that FSH signaling is involved in pathogenesis of menopausal depression, and likely to maintain the redox-optimized ROS balance in neurons.

Keywords: FSH; aging; antioxidants; metabolism; ROS; depression

Acta Pharmacologica Sinica (2020) 41:1033-1040; https://doi.org/10.1038/s41401-020-0384-8

\section{INTRODUCTION}

Major depressive disorder is a main cause of disability that severely diminishes psychosocial functioning and affects quality of life [1]. Clinical studies have demonstrated an increased risk of depression, accompanied by dramatic changes in reproductive hormones during the menopause transition [2]. The waning neuroprotective effect of estrogen is recognized as a main cause of menopausal depression [3, 4]. However, the effectiveness of estrogen replacement therapy, as part of the therapeutic armamentarium for menopausal depression, is still controversial $[5,6]$. Several large clinical trials such as the Women's International Study of Long-Duration Estrogen after the Menopause, and the Women's Health Initiative Study of Cognitive Aging, have demonstrated that estrogen therapy does not have an effect on mood regulation in postmenopausal women $[7,8]$. These studies suggest that other hormones are involved in the risk of menopausal depression. Thus, it is worthwhile to investigate the role of other reproductive hormones, including follicle-stimulating hormone (FSH), in the development of menopausal depression.

$\mathrm{FSH}$, a gonadotropin that triggers estrogen production, has been described to have significant extragonadal physiological functions, including promoting hepatic gluconeogenesis [9] and adipocytic lipid biosynthesis [10]. Higher FSH levels in the menopausal transition are associated with changes in brain function, such as sleep disturbance [11]. However, the association between FSH levels and mood disorders, including menopausal depression, is still unclear. Indeed, studies have reported that FSH levels in postmenopausal depressed women are higher, lower or unchanged compared with those in control women [12-14]. These contradictory results have driven us to investigate whether and how FSH affects menopausal depression in women.

FSH stimulates glutathione synthesis to increase antioxidant defense in rat/mouse ovarian follicles and granulosa cells, which are sensitive to oxidative stress-related damages [15-17]. The literatures have shown that oxidative stress plays a fundamental role in the etiology of depression $[18,19]$. In the current study, we found that $\mathrm{Fshr}^{-1-}$ mice exhibited more severe oxidative stress in the whole brain and increased depression-like behaviors, which were abrogated by the ROS scavenger NAC. Moreover, FSH signaling regulated the ROS balance by upregulating glutathione synthesis and promoting the NADP/NADPH transition in the phosphate pentose pathway. These findings reveal a novel role of FSH signaling in pathogenesis of menopausal depression in women.

\section{MATERIALS AND METHODS}

Animal experiments

Eight-week-old female C57/B6 $\mathrm{Fshr}^{-/-}$and $\mathrm{Fshr}^{+/+}$mice (Wuhan Kangweida Gene Technology Company, China) were subjected to ovariectomy. The mice were housed at $24^{\circ} \mathrm{C}$ on a $12: 12 \mathrm{~h}$ light/ dark cycle and provided standard chow and water ad libitum.

\footnotetext{
${ }^{1}$ Department of Endocrinology, Shandong Provincial Hospital Affiliated to Shandong University, Ji-nan 250000, China; ${ }^{2}$ Shandong Provincial Key Laboratory of Endocrinology and Lipid Metabolism, Ji-nan 250000, China; ${ }^{3}$ Institute of Endocrinology and Metabolism, Shandong Academy of Clinical Medicine, Ji-nan 250000 , China; ${ }^{4}$ School of Medicine, Shandong University, Ji-nan 250000, China; ${ }^{5}$ Department of Breast Thyroid Surgery, Shandong Provincial Hospital Affiliated to Shandong University, Ji-nan 250000, China;

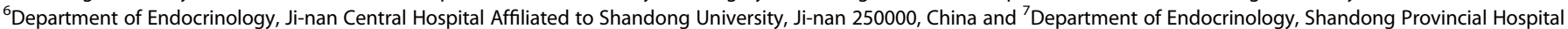
Affiliated to Shandong First Medical University and Shandong Academy of Medical Sciences, Ji-nan 250000, China Correspondence: Ling Gao (linggao@sdu.edu.cn) or Jia-jun Zhao (jjzhao@sdu.edu.cn) or Zhao He (zhaohe7711@qq.com)
}

Received: 15 October 2019 Accepted: 19 February 2020

Published online: 18 March 2020 
Animal experiments were approved by the Ethics Committee of Shandong Provincial Hospital Affiliated to Shandong University. After ovariectomy, NAC (150 mg/kg weight) or vehicle (saline) was administered i.p. to $\mathrm{Fshr}^{-1-}$ and $\mathrm{Fshr}^{+/+}$mice every day for 12 consecutive weeks.

\section{Behavior test}

(1) Forced swim test (FST). A mouse was placed in a plastic cylinder $\left(45 \mathrm{~cm}\right.$ high $\times 20 \mathrm{~cm}$ in diameter) filled with water $\left(23-24^{\circ} \mathrm{C}\right.$; $28-30 \mathrm{~cm}$ deep), and allowed to swim freely. Immobility was defined as floating without movements other than those necessary for the mouse to balance its body and keep its head above the water. Immobility time was quantified during the last $4 \mathrm{~min}$ of the 6 min test by the SMART system (Panlab Harvard Apparatus, Barcelona, Catalonia, Spain) [20].

(2) Elevated plus-maze (EPM) test. The maze was cleaned with $70 \%$ ethanol before each mouse was tested. Then, a mouse was placed in the center square $(10 \mathrm{~cm} \times 10 \mathrm{~cm})$ of the maze facing one of the open arms ( $40 \mathrm{~cm}$ above the floor). The number of entries and time spent in both open arms $(30 \mathrm{~cm} \times 5 \mathrm{~cm})$ and the enclosed arms $(30 \mathrm{~cm} \times 5 \mathrm{~cm}$ with $15 \mathrm{~cm}$-high walls) were recorded by the SMART system (Panlab Harvard Apparatus) for $5 \mathrm{~min}$ [20]. The percentages of open arm entries and time spent in the open arms were calculated as the number of open arm entries divided by the total number of arm entries and the time spent in the open arms divided by the total time, respectively [21].

(3) Tail-suspension test (TST). Mice were suspended individually by the tail with adhesive tape as described previously [21]. The duration of immobility over the 6 min test period was recorded. Immobility was defined as when the mouse hung passively and motionless, and immobility time was quantified during the last 4 min of the 6 min test by the SMART system (Panlab Harvard Apparatus) [20].

(4) Sucrose preference test. Mice were individually habituated to a bottle containing $1 \%$ sucrose and a second bottle containing water on either side of the cage, and the placement of the bottles was changed every day. During the first 3 days, baseline sucrose and water intake were recorded. After deprivation of both food and water for $24 \mathrm{~h}$, the mice were exposed to a bottle containing sucrose and a bottle containing water, but not food, for $12 \mathrm{~h}$. Sucrose preference was expressed as (sucrose/(sucrose + water)) $\times$ $100 \%$ [22].

(5) Morris water maze (MWM). The maze was a circular pool (120 $\mathrm{cm}$ in diameter) filled with water $\left(22-23^{\circ} \mathrm{C} ; 50 \mathrm{~cm}\right.$ in depth) that was made opaque with nontoxic Crayola paint. The maze was divided into four quadrants (southwest, southeast, northwest, and northeast). For hidden platform training, a $10 \mathrm{~cm}$ diameter platform was placed $25 \mathrm{~cm}$ from the wall and $2 \mathrm{~cm}$ below the surface of the water in the center of the southwest quadrant. The mice received four training sessions (entry locations: north, south, east, and west) daily for 5 consecutive days. The maximum time allowed per training session was $60 \mathrm{~s}$. For the probe trial on day 6 , the platform was removed and the mice were placed in the northeast quadrant and allowed to swim freely for $60 \mathrm{~s}$. The time spent in each quadrant, the latency to reach the platform, the distance traveled to reach the platform and mean swim speed were quantified by the SMART system (Panlab Harvard Apparatus).

(6) Open field test (OFT). The OFT was a white Plexiglas box $(40 \mathrm{~cm} \times 40 \mathrm{~cm} \times 40 \mathrm{~cm})$, and the mice were allowed to move freely in the box for $6 \mathrm{~min}$. The total distance traveled by the mice was recorded by the SMART system (Panlab Harvard Apparatus) [23].
(7) Rotarod test. The rotarod test was used to evaluate the activity of the mice and was performed according to previous studies [24].

\section{Immunostaining}

The primary antibodies were used in this study: FSHR (OM188095, OmnimAbs, Alhambra, CA, USA), NeuN (ab104224, Abcam, Cambridge, MA, USA), GFAP (66190-1-lg, Proteintech, Wuhan, China). Then, a corresponding secondary antibody was incubated for $1 \mathrm{~h}$ at room temperature. After washing, immunostaining was detected using a fluorescence microscope (Image A2 Zeiss, Oberkochen, Ostalbkreis, Germany).

\section{Immunoblotting}

Protein was extracted from hippocampal tissues or N2a cells, separated by $10 \%$ SDS-PAGE and transferred to PVDF membranes (Merck Millipore, Darmstadt, Germany). After incubation for $1 \mathrm{~h}$ with $5 \%$ nonfat milk in TBST at room temperature, the following primary antibodies were used: G6PD (12263, CST, Danvers, MA, USA), GCLM (14241-1-AP, Proteintech, Wuhan, China), GCLC (12601-1-AP, Proteintech), and $\beta$-actin (60008-1, Proteintech). Then membranes were incubated with secondary antibodies at room temperature for $1 \mathrm{~h}$. Finally, the immune complexes were detected by chemiluminescence.

\section{Enzyme immunoassay}

A mouse estradiol-sensitive ELISA kit (DE4399, Demeditec, Kiel, Germany), a testosterone ELISA kit (csb-E05101m, Cusabio, Wuhan, China), and an FSH ELISA kit (KA2330, Abnova, Taiwan, China) were used to measure the concentrations of estradiol, testosterone, and FSH in the serum according to the manufacturer's instructions.

\section{GSH assay}

The GSH assay (S0053, Beyotime, Shanghai, China) was performed according to the manufacturer's instructions. GSH concentrations were calculated as the total glutathione concentration minus two times the GSSG concentration. After the GSH or GSSG concentration was divided by the amount of total protein, the GSH/GSSG ratio was calculated.

\section{Cell culture and treatment}

Measurement of cellular ROS by Mitosox. N2a cells were purchased from Shanghai Institutes for Biological Sciences and cultured in high-glucose DMEM (11965-092, Gibco, Waltham, MA, USA) supplemented with $10 \%$ fetal bovine serum. N2a cells grown on glass-bottom dish (D35-10-1-N, Invitrogen, Waltham, MA, USA) were pretreated with or without $50 \mathrm{ng} / \mathrm{mL}$ FSH (HOR253, Prospec, Rehovot, Israel) for $2 \mathrm{~h}$ and then exposed to $200 \mu \mathrm{M} \mathrm{H}_{2} \mathrm{O}_{2}$ for $1 \mathrm{~h}$. Mitochondria and nuclei were stained with MitoTracker (green, 1:2500, Beyotime, Shanghai, China) and Hoechst (blue, 1:2500, Beyotime, Shanghai, China), respectively. ROS was detected by Mitosox (red, 1:1000, Invitrogen, Waltham, MA, USA). The glassbottom dishes were washed with PBS and observed using a laser confocal-scanning microscope (SM 780, Zeiss, Oberkochen, Germany). The fluorescence intensity of cellular ROS was analyzed by ImageJ software, and two investigators randomly chose 10-15 cells from each group for calculation.

Mitochondrial respiration and glycolysis

The effects of FSH on mitochondrial respiration and glycolysis in N2a cells were evaluated by an XF96 Extracellular Flux analyzer (Seahorse Bioscience, N Billerica, MA USA), which measured the oxygen consumption rate (OCR) and extracellular acidification rate (ECAR). Cells were plated at a density of $1 \times 10^{5}$ cells/well in a 96well culture plates and incubated in growth medium for $24 \mathrm{~h}$. Then the cells were treated with FSH $(50 \mathrm{ng} / \mathrm{mL})$ or vehicle for $12 \mathrm{~h}$. Then, a mitochondria stress test and glycolysis stress test 


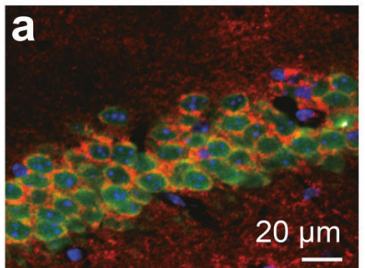

\section{FSHR/NeuN/DAPI}
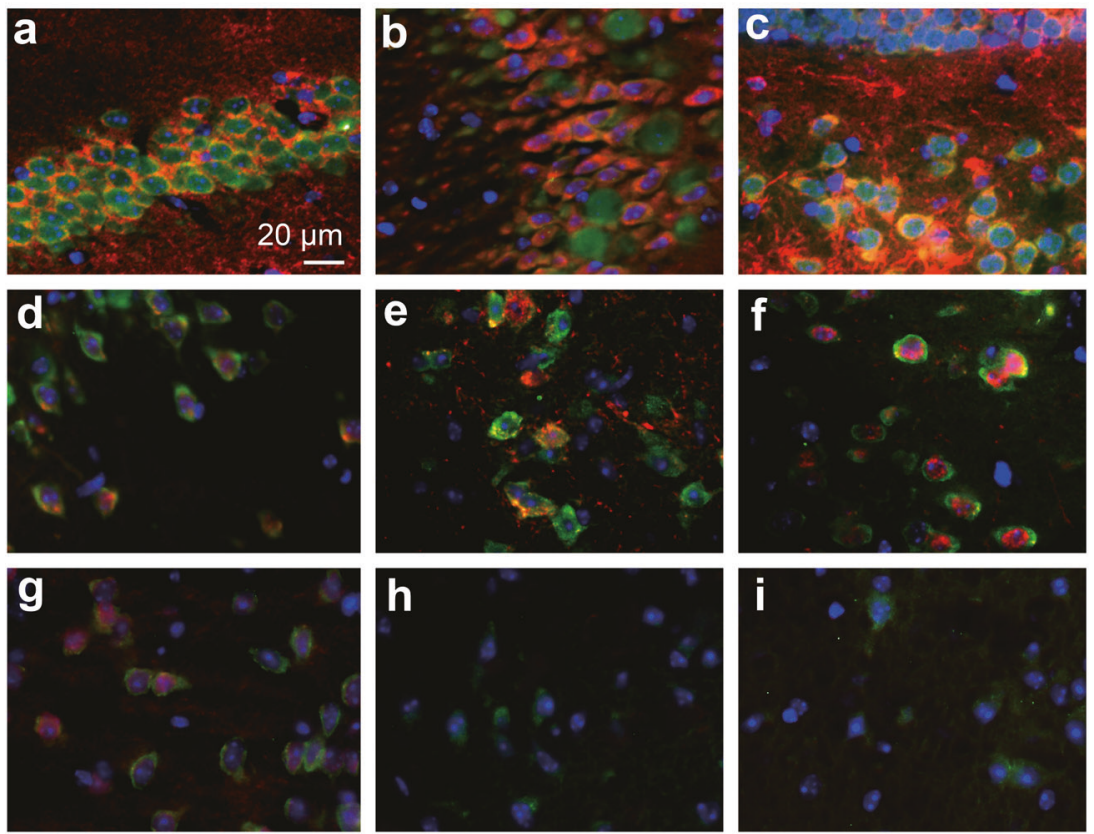

Fig. 1 The expression of FSHR in depression-related brain regions. a The CA1 region of the mouse hippocampus. $\mathbf{b}$ The CA3 region of mouse hippocampus. c The DG of the mouse hippocampus. $\mathbf{d}$ The cortex. e The nucleus accumbens. $\mathbf{f}$ The amygdala. $\mathbf{g}$ The prefrontal cortex. $\mathbf{h}$ The bed nucleus of the stria terminalis. i The lateral habenula. FSHR (red), NeuN (green, a marker of neurons), and DAPI (blue) immunofluorescence was detected under a fluorescence microscope

were performed to assess the key parameters of mitochondrial function and glycolytic flux according to the manufacturer's instructions. The OCR and ECAR values of each well were normalized by the protein content. Data were analyzed by Seahorse XF Report Generator (Seahorse Bioscience, N Billerica, MA USA).

Statistical analysis

Continuous variables are expressed as the means \pm standard deviations (SD). The differences between two groups were determined by Student's $t$ test. Differences among multiple groups were detected using one-way ANOVA. $P<0.05$ was considered statistically significant.

\section{RESULTS}

FSHR expression in depression-related brain regions

To investigate the role of FSH signaling in depression, we first determined the expression of FSHR in brain regions that control depression-like behaviors. The immunofluorescence results showed that FSHR was present in neurons, but not astrocytes (Fig. S1), in multiple depression-related regions, including the hippocampus, cortex, nucleus accumbens, amygdala, and prefrontal cortex (Fig. 1a-g), not in the bed nucleus of the stria terminalis or lateral habenula (Fig. 1h, i). Neurons in these regions are closely related to the occurrence of depression [25-27].

Ablation of FSHR leads to depression-like behaviors

To explore whether FSH signaling is involved in the development of menopausal depression, we performed the FST, EPM, TST, and sucrose preference test to assess depression-related behavior in postmenopausal (ovariectomized) FSHR-null mice and found that $\mathrm{Fshr}^{-1-}$ mice exhibited a longer immobility time than control $\mathrm{Fshr}^{+/+}$mice in the FST, indicating increased vulnerability to menopausal depression (Fig. 2a). No differences were found in the sucrose preference test, EPM, TST, and MWM (Fig. 2b-j). To rule out the possibility that the longer immobility time of FSHR knockout mice in the FST was due to general motor deficits, we investigated spontaneous activity by the open field test and assessed forced activity by the rotarod test. No difference was detected between FSHR knockout and wild-type mice in these two tests (Fig. 2k, I), suggesting that the immobility of FSHR knockout mice was likely due to depression-like behavior. Previous studies have reported that both estrogen and androgen are involved in the regulation of depression [28, 29]. To address this issue of the depression-like behaviors were possibly caused by the change of these hormones in $\mathrm{Fshr}^{-/-}$mice, we next examined serum estradiol and testosterone levels in mice and found no significant difference in serum estradiol, testosterone or FSH levels or body weight between ovariectomized $\mathrm{Fshr}^{-/-}$and $\mathrm{Fshr}^{+/+}$mice (Fig. S2). These results indicated that the depression-like behavior of $\mathrm{Fshr}^{-1-}$ mice is indeed, at least in part, due to the absence of FSH signaling.

FSHR deficiency increases cellular oxidative stress

Recent studies have found that oxidative stress is associated with the development of depression, including DNA damage, lipid peroxidation, and enzyme inactivation [19]. Oxidative stress is a form of ROS imbalance in which ROS production overwhelms endogenous antioxidant systems including GSH levels. The GSH precursor NAC, an ROS scavenger, ameliorates depression-like behaviors in diabetic rats [30] and stress-induced depression in mice [31]. In rat and mouse ovarian follicles and granulosa cells, glutathione synthesis is increased by FSH stimulation, and ROSinduced apoptosis is thereby reduced [16]. Indeed, we found that FSHR knockout mice displayed more severe oxidative stress, as the GSH/GSSG ratio of the whole brains of $\mathrm{Fshr}^{-1-}$ mice was much lower than that of control mice (Fig. 3a). Next, we injected NAC into $\mathrm{Fshr}^{-1-}$ and control mice daily for 12 weeks and found that the administration of the ROS scavenger NAC improved the depression-like behavior of $\mathrm{Fshr}^{-1-}$ mice in the FST (Fig. 3b) and enhanced GSH/GSSG ratio in the whole brain (Fig. 3c). Therefore, these results demonstrated that oxidative stress is at least partly responsible for the development of depression-like behavior in $\mathrm{Fshr}^{-1-}$ mice. 

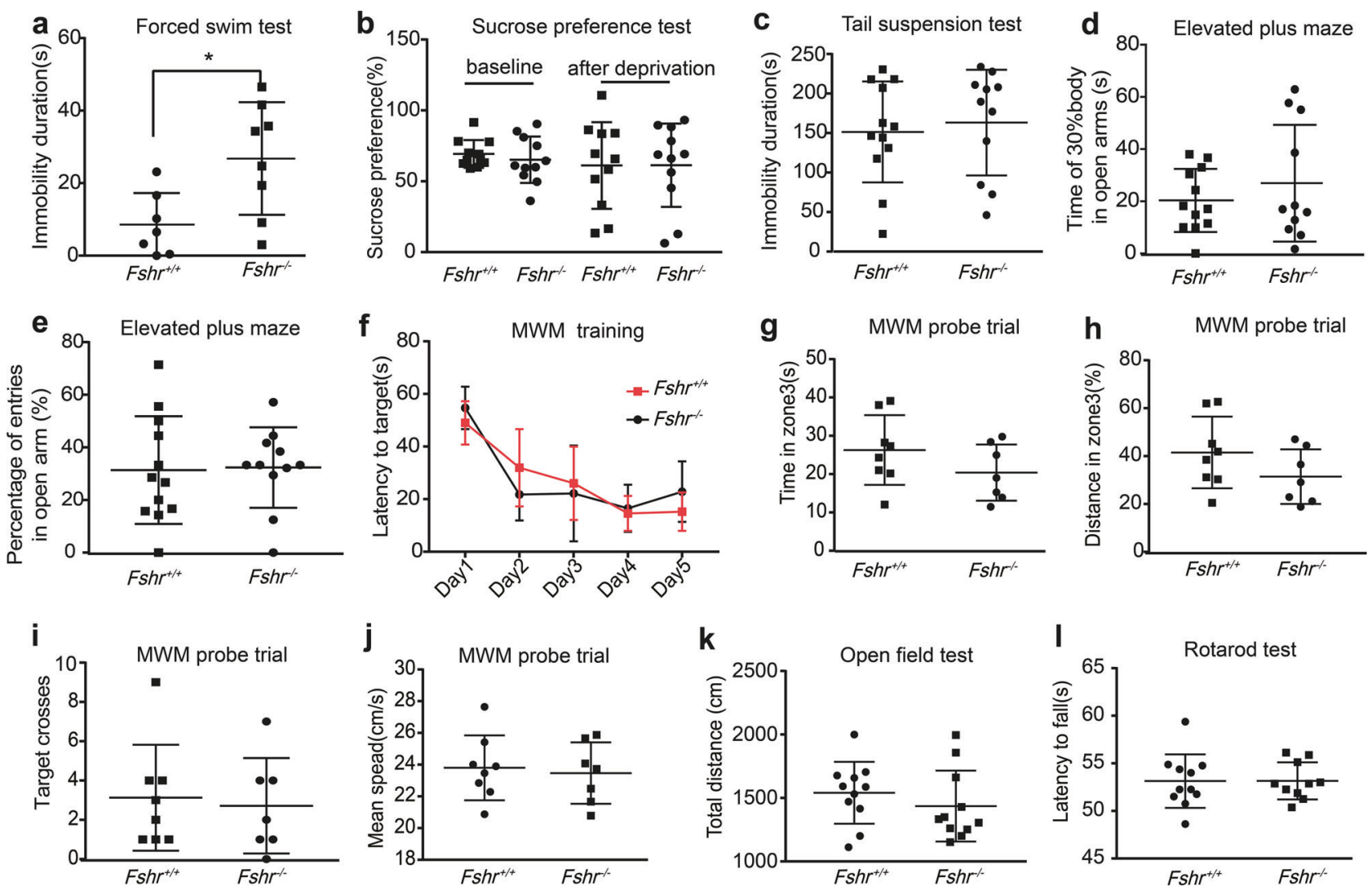

Fig. $2 \mathrm{Fshr}^{-1-}$ mice display depression-like behavior. a The immobility duration in the forced swim test. b Sucrose preference at baseline and after deprivation in the sucrose preference test. c The immobility duration in the tail-suspension test. Performance in elevated plus-maze, including time during which $30 \%$ the body was in an open arm (d) and the percentage of entries into the open arms (e). $\mathbf{f}$ The latency to reach the target in the training phase of the Morris water maze (MWM). Performance in the MWM probe trail, including latency (g), distance traveled in zone 3 (h), target crosses (i), and mean speed (j). $\mathbf{k}$ The total distance traveled in the open field test. I The latency to fall in the rotarod test. Each mouse is represented by a dot in the scatter plot, and the data are shown as the mean \pm SD. ${ }^{*} P<0.05$

Next, we used FSH to pretreat N2a cells, a mouse neuroblastoma cell line expressing FSHR (Fig. S3), and then induced cellular oxidative stress by the addition of hydrogen peroxide to observe the protective effect of FSH on cellular oxidative stress. The Mitosox fluorescence intensity, which reflects cellular ROS levels, showed that hydrogen peroxide-induced ROS levels were reversed by the addition of $\mathrm{FSH}$, suggesting that $\mathrm{FSH}$ improves cellular oxidative stress (Fig. 3d, e). Together, these results indicated that FSH-FSHR deficiency is associated with oxidative stress in neurons and depression-like behaviors.

FSH promotes glutathione synthesis and the phosphate pentose pathway (PPP)

To investigate how FSH-FSHR signaling regulates the ROS balance, we next evaluated GSH metabolism, including glutathione synthesis and the PPP. Indeed, the addition of FSH increased the protein level of glutamate cysteine ligase modifier subunit (GCLm), a rate-limiting enzyme of GSH synthesis, without altering the protein level of glutamate cysteine ligase catalytic subunit (GCLC) in vitro (Fig. 4a, b). Conversely, FSHR deletion reduced GCLm protein levels, but not GCLc protein levels (Fig. 4c, d). These results suggested that FSH-FSHR signaling regulates glutathione synthesis in neurons.

The PPP, a basic NADPH-producing pathway, regulates ROS balance by controlling the content of GSH. The protein level of glucose-6-phosphate dehydrogenase (G6PD) (Fig. 4e, f) was dramatically enhanced by FSH supplementation, suggesting that the elevation of the PPP enhances NADPH production. Consistent with these observations, the genetic ablation of FSHR decreased the protein level of G6PD in vivo (Fig. $4 \mathrm{~g}, \mathrm{~h}$ ). Together, these data suggested that FSH regulates GSH metabolism by enhancing the rate-limiting enzyme of glutathione synthesis and the PPP.

In addition, the PPP is one of the pathways that regulate glucose metabolism, which is closely related to ROS balance [32]. To further investigate the role of FSH in ROS balance, the two other glucose metabolism pathways, mitochondrial respiration and glycolysis, were evaluated in neurons. FSH-treated N2a cells showed reductions in the basal mitochondrial respiration rate, the maximal respiration rate, and ATP production, suggesting that mitochondrial respiration was inhibited (Fig. 4i, j). The glycolysis rate and glycolytic reserve were lower in FSH-treated N2a cells. The elevated ECAR induced by oligomycin was also suppressed by FSH supplementation, demonstrating that glycolytic capacity was inhibited by $\mathrm{FSH}$ (Fig. 4k-m). The inhibition of mitochondrial respiration and glycolysis by FSH supplementation is likely due to the enhancement of the PPP, as FSH promotes the diversion of glucose into the PPP but not mitochondrial respiration or glycolysis. Together, these data supported the hypothesis that FSH upregulates the PPP to mediate ROS balance.

\section{DISCUSSION}

In the current study, FSH was shown to be involved in the pathogenesis of menopausal depression and mediate redoxoptimized ROS balance in the central nervous system. FSHR ablation reduced GCLm and G6PD protein levels, leading to oxidative stress and thus depression-like behaviors. Consistently, FSH increased GCLm and G6PD protein levels and decreased ROS 

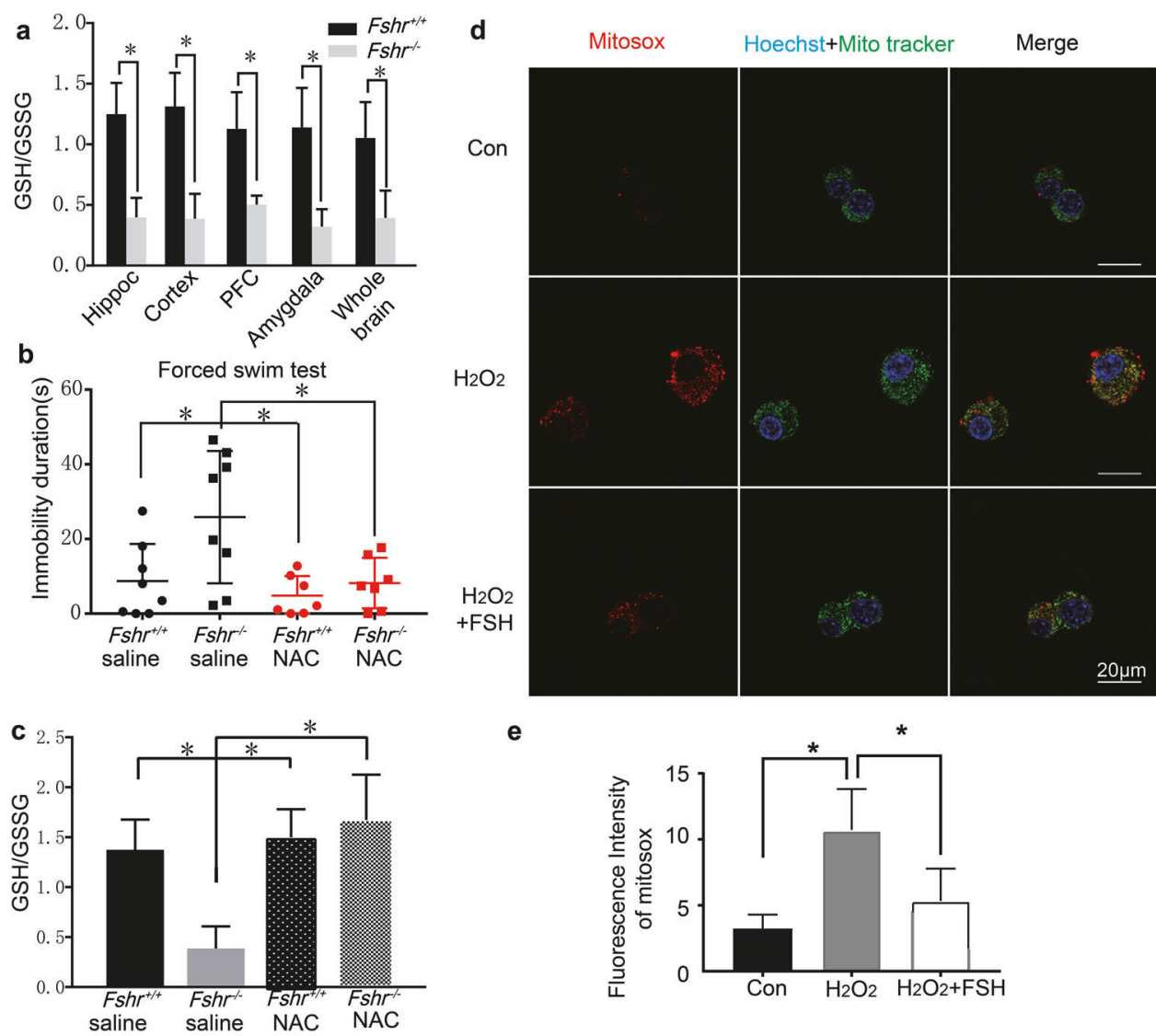

e

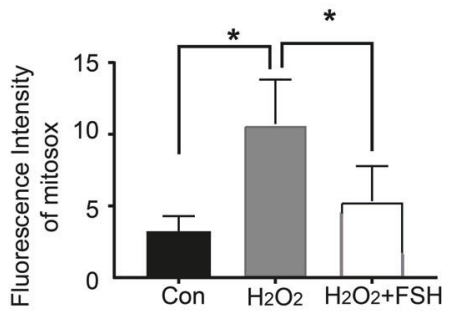

Fig. 3 FSHR ablation induces cellular ROS imbalance. a The GSH/GSSG ratio in brain tissues from Fshr ${ }^{+/+}$and Fshr ${ }^{-/-}$mice. The data are shown as the mean \pm SD. ${ }^{*} P<0.05, n=7-8$ per group. $\mathbf{b}$ The immobility duration in the forced swim test. Each mouse is represented by a dot in the scatter plot, and the data are shown as the mean \pm SD. ${ }^{*} P<0.05$. c The GSH/GSSG ratio in the whole brains of Fshr ${ }^{+/+}$and Fshr ${ }^{-/-}$mice treated with NAC or vehicle. The data are shown as the mean \pm SD. ${ }^{*} P<0.05, n=7-8$ per group. d Mitosox, Hoechst, and MitoTracker staining in N2a cells. e The fluorescence intensity of Mitosox in N2a cells. The data are shown as the mean \pm SD. ${ }^{*} P<0.05, n=12-15$ per group

production. These findings provide preclinical evidence that the alteration of reproductive hormone profiles is closely associated with an increased risk of depression in women during the menopausal transition. The induction of depression-like behaviors by FSHR inactivation, implies that lower serum FSH levels during the menopause transition are likely to be associated with the occurrence of menopausal depression in women. Consistent with our results, several studies have reported that gonadotropin levels in postmenopausal depressed women are lower than those in controls $[13,33]$. Conversely, higher FSH levels have been found to be associated with negative mood in women during the menopausal transition [12, 34]. The Study of Women's Health Across the Nation found that estrogen and FSH levels are not associated with depression, and cannot account for the correlation of menopausal status with depression [29]. A recent preclinical study found that stress-induced depressive rats display higher FSH levels than control rats and presumed that increased FSH levels might be the cause of lower monoamine release [35]. These contradictory results imply that menopausal depression is caused by a variety of risk factors such as genetic background, race, lifestyle, and stress events. In the present study, we found that the alteration of $\mathrm{FSH}$ signaling is associated with menopausal depression, likely through the regulation of ROS balance, which provides novel preclinical insight into menopausal depression.

This research reveals the vital role of FSH signaling in redoxoptimized ROS balance, which is involved in the pathogenesis of depression throughout life. Consistently, congenital hypogonadotropic hypogonadism $(\mathrm{CHH})$ caused by FSH deficits induces depression even before the menopause transition. Research from
Sweden has shown that women with $\mathrm{CHH}$ are more likely to display depressive symptoms than women with common female factor infertility [36], indicating that the FSH signaling affects mood throughout life. Likewise, there is an increased frequency of anxiety and depression in young male patients with $\mathrm{CHH}$ [37]. Although the mechanism underlying depression in $\mathrm{CHH}$ patients is unclear, the increased morbidity of depression in $\mathrm{CHH}$ patients implies that FSH signaling is possibly involved in depression development during not only the menopause transition but also throughout life. Thus, further experiments are required to elucidate the effect of FSH signaling on mood in different phases of life, including puberty and mild age. In addition to oxidative stress, brain developmental disorders might be another possible cause of depression-like behaviors of patients with $\mathrm{CHH}$ and FSHR knockout mice. Therefore, inactivation of FSHR at different times is required to dissect the role of FSH signaling in depression during different phases of life.

FSH plays a vital role in energy metabolism. Higher serum FSH levels enhance hepatic gluconeogenesis [9] and lipid biosynthesis [10]. Blocking FSH with an FSH $\beta$ antibody or by FSHR inactivation induces thermogenic adipose tissue, reduces body fat [38], and conserves bone mass [39], providing a pharmacological target for treating menopause-related diseases. However, decreased cholesterol biosynthesis, which is induced by FSH signaling blockade [40], causes demyelination in the central nervous system [41]. Given that demyelination contributes to the initiation of depression [42], the lipid dysmetabolism induced by FSHR inactivation is a possible cause of the depression-like behaviors of $\mathrm{Fshr}^{-1-}$ mice. Hence, neuron-specific FSHR knockout mice are required to 

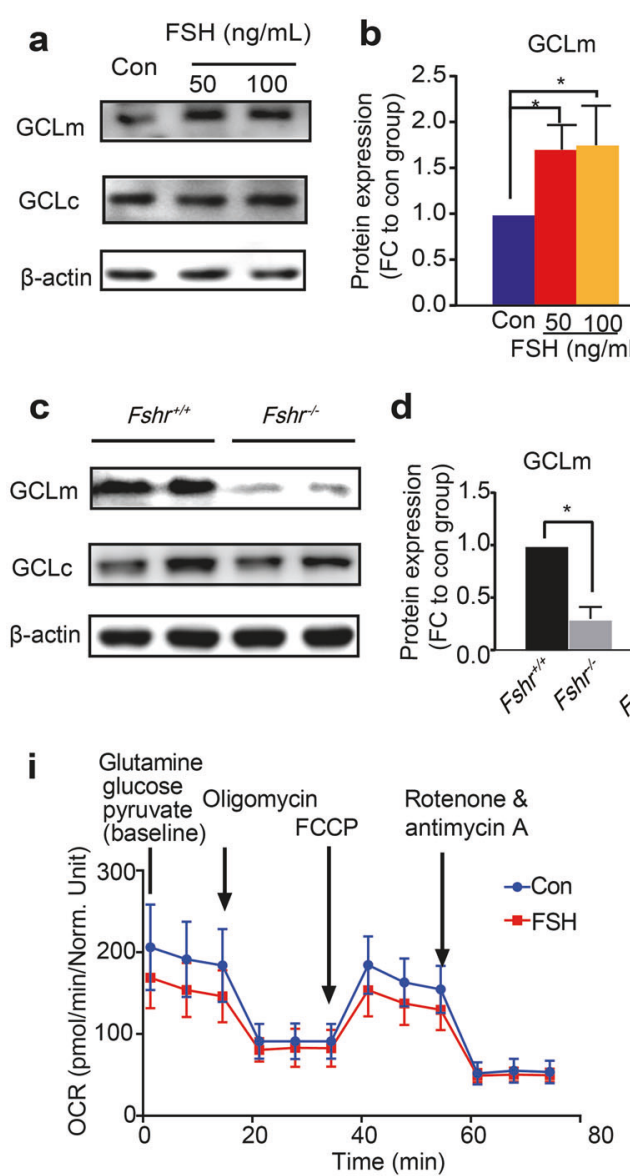

k

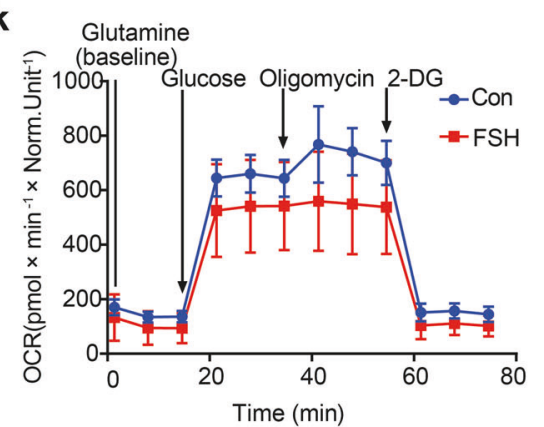

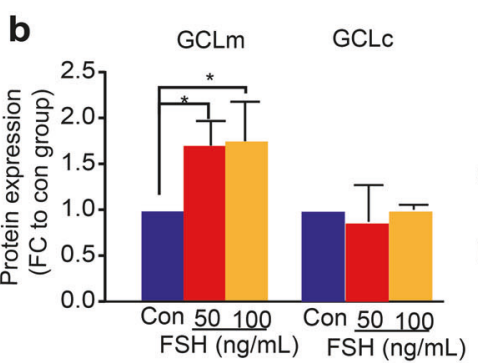

d

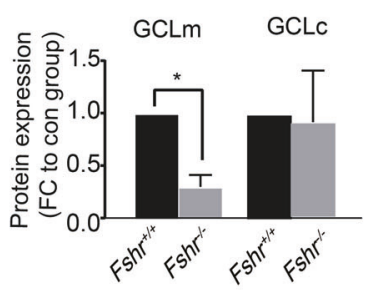

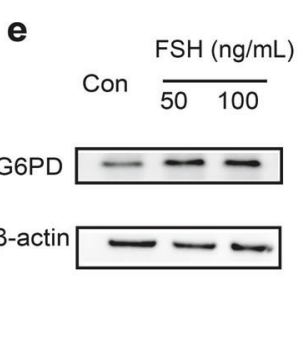

g

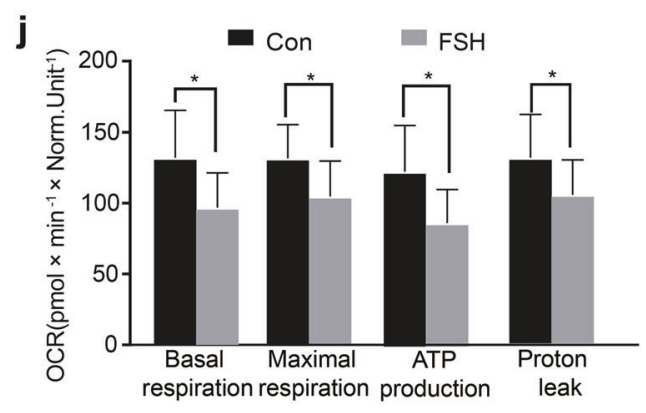

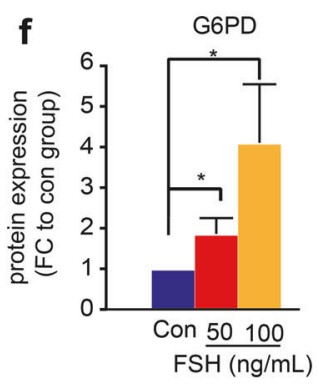

h

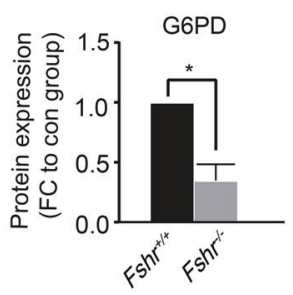

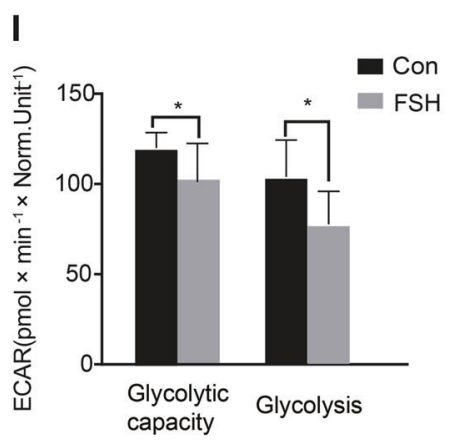

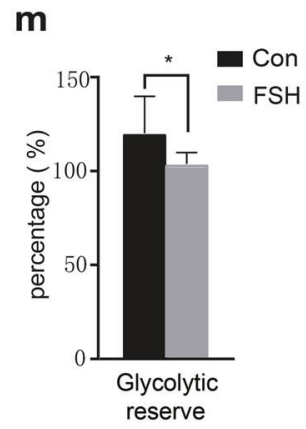

Fig. 4 FSH regulates GSH metabolism through glutathione synthesis and the phosphate pentose pathway. $\mathbf{a}$, $\mathbf{b}$ The protein expression of the modifier subunit (GCLm) and glutamate cysteine ligase catalytic subunit (GCLc) in N2a cells treated with or without FSH for 2 h. GCLm and GCLc were detected by Western blot analysis (a). Grayscale values of protein expression were normalized to those of the control group (b). The protein expression of GCLm and GCLc in hippocampal tissues from Fshr ${ }^{+/+}$and Fshr ${ }^{-1-}$ mice. GCLm and GCLc were detected by Western blot analysis (c), and the grayscale values of protein expression were normalized to those of $F$ shr ${ }^{+/+}$mice (d). The protein expression of G6PD in $\mathrm{N} 2 \mathrm{a}$ cells treated with FSH $(50 \mathrm{ng} / \mathrm{mL})$ or vehicle. G6PD was detected by Western blot analysis (e), and the grayscale values of protein expression were normalized to those of the control group (f). $\mathbf{g}, \mathbf{h}$ The protein expression of G6PD in hippocampal tissues from Fshr ${ }^{+/+}$and $\mathrm{Fshr}^{-/-}$mice. G6PD was detected by Western blot analysis (g). The grayscale values of protein expression were normalized to those of $F$ shr $r^{+/+}$ mice (h). i A kinetic graph of mitochondrial stress tests in N2a cells treated with $\mathrm{FSH}(50 \mathrm{ng} / \mathrm{mL})$ or vehicle. $\mathbf{j}$ Basal mitochondrial respiration, maximal respiration capacity, ATP production, and proton leak were measured under mitochondrial stress. The values represent the mean \pm SD. ${ }^{*} P<0.05, n=10-15$ per group. k A kinetic graph of glycolytic stress tests in N2a cells treated with FSH (50 ng/mL) or vehicle. Glycolytic capacity, glycolysis (I), and glycolytic reserve $(\mathbf{m})$ measured by glycolytic stress tests. The data are shown as the mean \pm SD. ${ }^{*} P<0.05, n=10-15$ per group

explain the effect of FSH on mood. Importantly, our study indicated that treating menopause-related diseases by blocking FSH signaling may lead to side effects, including an increased risk of depression.

Emerging evidence has indicated that excess ROS play a pivotal role in the pathogenesis of depression. The protein level of GSH, a major antioxidant, is decreased in the brains of depressed patients
[43] and stress-induced rats [44]. In our study, $\mathrm{Fshr}^{-1-}$ mice showed a lower GSH/GSSG ratio, indicating more severe oxidative stress in the absence of FSHR. GSH prevents stress-induced depression-like behaviors [44]. NAC, the precursor of GSH and an ROS scavenger, attenuates the depression-like behavior of diabetic rats and stress-induced depressive mice. Behavior changes in diabetic rats and stress-induced depressive mice 
induced by NAC administration are associated with a reduction in oxidative stress in the hippocampus [30,31]. Consistent with these studies, $\mathrm{Fshr}^{-1-}$ mice displayed depression-like behaviors that were attenuated by NAC, suggesting that oxidative stress, at least partially, induced depression-like behaviors. To better understand the depression-like behaviors of $\mathrm{Fshr}^{-/-}$mice, further studies are required to elucidate how the absence of FSHR causes depression.

In conclusion, this study showed that FSHR inactivation induces depression-like behaviors, providing direct evidence that FSH signaling mediates the development of postmenopausal depression and the side effects of FSH signaling blockade. Moreover, FSH exerts potent antioxidant effects by regulating GSH metabolism, providing novel insight into the pathophysiology of menopauserelated disease.

\section{ACKNOWLEDGEMENTS}

This work was supported by the National Key Research and Development Program of China (2017YFC1309800), National Natural Science Foundation of China (81430020, 81670796, 31471321, and 81900716), Key Research and Development Plan of Shandong province (2017G006006), and Shandong Provincial Natural Science Foundation (ZR2019BH023). We acknowledge the support of Yan-Meng Zhou for the behavior tests.

\section{AUTHOR CONTRIBUTIONS}

WKB performed the experiments and analyzed the data; SSS, ZWL, YWR, SSL, and TG performed the experiments; WKB wrote the paper; ZH, JJZ, and LG conceived the idea; and ZHD, JW, SSW, and SZM assisted with animal studies. All authors read and approved the final paper.

\section{ADDITIONAL INFORMATION}

The online version of this article (https://doi.org/10.1038/s41401-020-0384-8) contains supplementary material, which is available to authorized users.

Competing interests: The authors declare no competing interests.

\section{REFERENCES}

1. Malhi GS, Mann JJ. Depression. Lancet. 2018;392:2299-312.

2. Soares CN. Depression and menopause: an update on current knowledge and clinical management for this critical window. Med Clin North Am. 2019;103: 651-67.

3. Frokjaer VG, Pinborg A, Holst KK, Overgaard A, Henningsson S, Heede M, et al. Role of serotonin transporter changes in depressive responses to sex-steroid hormone manipulation: a positron emission tomography study. Biol Psychiatry. 2015;78:534-43.

4. Jovanovic H, Kocoska-Maras L, Radestad AF, Halldin C, Borg J, Hirschberg AL, et al. Effects of estrogen and testosterone treatment on serotonin transporter binding in the brain of surgically postmenopausal women-a PET study. Neuroimage. 2015;106:47-54.

5. Joffe $H$, Petrillo LF, Koukopoulos A, Viguera AC, Hirschberg A, Nonacs $R$, et al. Increased estradiol and improved sleep, but not hot flashes, predict enhanced mood during the menopausal transition. J Clin Endocrinol Metab. 2011;96: E1044-54.

6. Morrison MF, Kallan MJ, Ten Have T, Katz I, Tweedy K, Battistini M. Lack of efficacy of estradiol for depression in postmenopausal women: a randomized, controlled trial. Biol Psychiatry. 2004;55:406-12.

7. Resnick SM, Coker LH, Maki PM, Rapp SR, Espeland MA, Shumaker SA. The Women's Health Initiative Study of Cognitive Aging (WHISCA): a randomized clinical trial of the effects of hormone therapy on age-associated cognitive decline. Clin Trials. 2004;1:440-50.

8. Welton AJ, Vickers MR, Kim J, Ford D, Lawton BA, MacLennan AH, et al. Health related quality of life after combined hormone replacement therapy: randomised controlled trial. BMJ. 2008;337:a1190.

9. Qi X, Guo Y, Song Y, Yu C, Zhao L, Fang L, et al. Follicle-stimulating hormone enhances hepatic gluconeogenesis by GRK2-mediated AMPK hyperphosphorylation at Ser485 in mice. Diabetologia. 2018;61:1180-92.

10. Liu XM, Chan HC, Ding GL, Cai J, Song Y, Wang TT, et al. FSH regulates fat accumulation and redistribution in aging through the Galphai/Ca(2+)/CREB pathway. Aging Cell. 2015;14:409-20.
11. Slopien R, Wichniak A, Pawlak M, Slopien A, Warenik-Szymankiewicz A, Sajdak S. Disturbances of sleep continuity in women during the menopausal transition. Psychiatr Pol. 2015;49:615-23.

12. Berent-Spillson A, Marsh C, Persad C, Randolph J, Zubieta JK, Smith Y. Metabolic and hormone influences on emotion processing during menopause. Psychoneuroendocrinology. 2017;76:218-25.

13. Brambilla F, Maggioni M, Ferrari E, Scarone S, Catalano M. Tonic and dynamic gonadotropin secretion in depressive and normothymic phases of affective disorders. Psychiatry Res. 1990;32:229-39.

14. Bromberger JT, Kravitz HM. Mood and menopause: findings from the Study of Women's Health Across the Nation (SWAN) over 10 years. Obstet Gynecol Clin North Am. 2011;38:609-25.

15. Hoang YD, Nakamura BN, Luderer U. Follicle-stimulating hormone and estradio interact to stimulate glutathione synthesis in rat ovarian follicles and granulosa cells. Biol Reprod. 2009;81:636-46.

16. Shen M, Jiang Y, Guan Z, Cao Y, Sun SC, Liu H. FSH protects mouse granulosa cells from oxidative damage by repressing mitophagy. Sci Rep. 2016;6:38090.

17. Tsai-Turton $M$, Luderer U. Opposing effects of glutathione depletion and folliclestimulating hormone on reactive oxygen species and apoptosis in cultured preovulatory rat follicles. Endocrinology. 2006;147:1224-36.

18. Farias JG, Herrera EA, Carrasco-Pozo C, Sotomayor-Zarate R, Cruz G, Morales P, et al. Pharmacological models and approaches for pathophysiological conditions associated with hypoxia and oxidative stress. Pharmacol Ther. 2016;158:1-23.

19. Michel TM, Pulschen D, Thome J. The role of oxidative stress in depressive disorders. Curr Pharm Des. 2012;18:5890-9.

20. Zhang HT, Huang Y, Jin SL, Frith SA, Suvarna N, Conti M, et al. Antidepressant-like profile and reduced sensitivity to rolipram in mice deficient in the PDE4D phosphodiesterase enzyme. Neuropsychopharmacology. 2002;27:587-95.

21. Gong MF, Wen RT, Xu Y, Pan JC, Fei N, Zhou YM, et al. Attenuation of ethanol abstinence-induced anxiety- and depressive-like behavior by the phosphodiesterase-4 inhibitor rolipram in rodents. Psychopharmacology. 2017; 234:3143-51.

22. Liu MY, Yin $\mathrm{CY}$, Zhu LJ, Zhu XH, Xu C, Luo CX, et al. Sucrose preference test for measurement of stress-induced anhedonia in mice. Nat Protoc. 2018;13:1686-98.

23. Bay-Richter C, Janelidze S, Sauro A, Bucala R, Lipton J, Deierborg T, et al. Behavioural and neurobiological consequences of macrophage migration inhibitory factor gene deletion in mice. J Neuroinflammation. 2015;12:163.

24. Kremer PM, Torres DJ, Hashimoto AC, Berry MJ. Disruption of selenium handling during puberty causes sex-specific neurological impairments in mice. Antioxidants. 2019;8:110. https://doi.org/10.3390/antiox8040110.

25. Kucharczyk M, Kurek A, Pomierny B, Detka J, Papp M, Tota K, et al. The reduced level of growth factors in an animal model of depression is accompanied by regulated necrosis in the frontal cortex but not in the hippocampus. Psychoneuroendocrinology. 2018;94:121-33.

26. Hodes A, Rosen $\mathrm{H}$, Cohen-Ben Ami H, Lichtstein D. $\mathrm{Na}^{+}, \mathrm{K}^{+}$-ATPase alpha3 isoform in frontal cortex GABAergic neurons in psychiatric diseases. J Psychiatr Res. 2019;115:21-8.

27. Lee AL, Ogle WO, Sapolsky RM. Stress and depression: possible links to neuron death in the hippocampus. Bipolar Disord. 2002;4:117-28.

28. Birkhauser M. Depression, menopause and estrogens: is there a correlation? Maturitas. 2002;41(Suppl 1):S3-8.

29. Bromberger JT, Schott LL, Kravitz HM, Sowers M, Avis NE, Gold EB, et al. Longitudinal change in reproductive hormones and depressive symptoms across the menopausal transition: results from the Study of Women's Health Across the Nation (SWAN). Arch Gen Psychiatry. 2010;67:598-607.

30. Reus GZ, Dos Santos MA, Abelaira HM, Titus SE, Carlessi AS, Matias BI, et al. Antioxidant treatment ameliorates experimental diabetes-induced depressivelike behaviour and reduces oxidative stress in brain and pancreas. Diabetes Metab Res Rev. 2016;32:278-88.

31. Fernandes J, Gupta GL. N-acetylcysteine attenuates neuroinflammation associated depressive behavior induced by chronic unpredictable mild stress in rat. Behav Brain Res. 2019;364:356-65.

32. Butterfield DA, Halliwell B. Oxidative stress, dysfunctional glucose metabolism and Alzheimer disease. Nat Rev Neurosci. 2019;20:148-60.

33. Amsterdam JD, Winokur A, Lucki I, Snyder P. Neuroendocrine regulation in depressed postmenopausal women and healthy subjects. Acta Psychiatr Scand. 1983;67:43-9.

34. Freeman EW, Sammel MD, Lin H, Gracia CR, Kapoor S. Symptoms in the menopausal transition: hormone and behavioral correlates. Obstet Gynecol. 2008;111: 127-36.

35. Gu S, Jing L, Li Y, Huang JH, Wang F. Stress induced hormone and neuromodulator changes in menopausal depressive rats. Front Psychiatry. 2018;9:253.

36. Dzemaili S, Tiemensma J, Quinton R, Pitteloud N, Morin D, Dwyer AA. Beyond hormone replacement: quality of life in women with congenital hypogonadotropic hypogonadism. Endocr Connect. 2017;6:404-12. 
37. Aydogan U, Aydogdu A, Akbulut H, Sonmez A, Yuksel S, Basaran Y, et al. Increased frequency of anxiety, depression, quality of life and sexual life in young hypogonadotropic hypogonadal males and impacts of testosterone replacement therapy on these conditions. Endocr J. 2012;59:1099-105.

38. Liu P, Ji Y, Yuen T, Rendina-Ruedy E, DeMambro VE, Dhawan S, et al. Blocking FSH induces thermogenic adipose tissue and reduces body fat. Nature. 2017;546: 107-12.

39. Sun L, Peng Y, Sharrow AC, Iqbal J, Zhang Z, Papachristou DJ, et al. FSH directly regulates bone mass. Cell. 2006;125:247-60.

40. Guo Y, Zhao M, Bo T, Ma S, Yuan Z, Chen W, et al. Blocking FSH inhibits hepatic cholesterol biosynthesis and reduces serum cholesterol. Cell Res. 2019;29:151-66.
41. Saher G, Stumpf SK. Cholesterol in myelin biogenesis and hypomyelinating disorders. Biochim Biophys Acta. 2015;1851:1083-94.

42. Ma T, Li B, Le $Y, X u$ Y, Wang F, Tian $Y$, et al. Demyelination contributes to depression comorbidity in a rat model of chronic epilepsy via dysregulation of Olig2/LINGO-1 and disturbance of calcium homeostasis. Exp Neurol. 2019;321: 113034.

43. Gawryluk JW, Wang JF, Andreazza AC, Shao L, Young LT. Decreased levels of glutathione, the major brain antioxidant, in post-mortem prefrontal cortex from patients with psychiatric disorders. Int J Neuropsychopharmacol. 2011;14:123-30.

44. Pal SN, Dandiya PC. Glutathione as a cerebral substrate in depressive behavior. Pharmacol Biochem Behav. 1994:48:845-51. 